\title{
Cross Sectional Imaging of Solitary Lesions of the Neurocranium
}

\section{Schnittbilddiagnostik solitärer Läsionen des Neurokraniums}

Authors

Max-Ludwig Schäfer ${ }^{1}$, Arend Koch ${ }^{2}$, Florian Streitparth ${ }^{1}$, Edzard Wiener ${ }^{3}$

Affiliations

1 Institute of Radiology, Charité university hospital Berlin, Campus Virchow-Clinic, Berlin, Germany

2 Institute of Neuropathology, Charité university hospital Berlin, Berlin, Germany

3 Institute of Neuroradiology, Charité university hospital Berlin, Berlin, Germany

Key words

$\mathrm{CT}, \mathrm{MR}$ imaging, solitary lesions of the neurocranium

received 16.12 .2016

accepted 23.07.2017

Bibliography

DOI https://doi.org/10.1055/s-0043-118481

Published online: 21.9.2017

Fortschr Röntgenstr 2017; 189: 1135-1144

(c) Georg Thieme Verlag KG, Stuttgart · New York

ISSN 1438-9029

Correspondence

Dr. Max-Ludwig Schäfer

Institut für Strahlenheilkunde, Charité Universitätsklinikum Berlin, Campus Virchow Klinikum, Augustenburger Platz 1, 13353 Berlin, Germany

Tel.: ++49/30/20787318

max_schaefer_001@hotmail.com

\section{ABSTRACT}

Background Although a wide range of processes along the neurocranium are of a benign nature, there are often difficulties in the differential diagnosis.

Method In the review CT/MRI scans of the head were evaluated retrospectively regarding solitary lesions along the neurocranium. The majority of the lesions were histologically proven.

Results The purpose of the review is to present typical pathologies of the neurocranium and provide a systematic overview based on 12 entities, their locations, prevalence and radiological characteristics.
Conclusion Processes, which primarily originate from the neurocranium have to be differentiated from secondary processes infiltrating the neurocranium. For this important diagnostic feature, MRI is typically essential, while the definitive diagnosis is often made on the basis of the medical history and the typical appearance on computer tomography.

\section{Key Points}

- There are often difficulties in the precise differential diagnosis of solitary lesions along the neurocranium. Typical solitary pathologies of the neurocranium based on 12 entities were presented. Both magnetic resonance imaging and computed tomography are often essential for an exact differential diagnosis.

\section{Citation Format}

- Schäfer M, Koch A, Streitparth F et al. Cross Sectional Diagnosis of Solitary Lesions of the Neurocranium. Fortschr Röntgenstr 2017; 189: 1135-1144

\section{ZUSAMMENFASSUNG}

Hintergrund Obgleich eine Vielzahl von Prozessen am Neurokranium Zufallsbefunde gutartiger Natur sind, gibt ist es dennoch häufig Schwierigkeiten bei der genaueren artdiagnostischen Einordnung.

Methode Im Rahmen der Übersichtsarbeit wurden retrospektiv CT/MRT des Schädels bezüglich solitärer Läsionen des Neurokraniums ausgewertet. Die Mehrzahl der Läsionen wurden histologisch gesichert.

Ergebnisse Ziel war es, typische lokale Pathologien des Neurokraniums darzustellen und anhand von 12 Entitäten, deren Lokalisationen, Häufigkeiten und radiologischen Besonderheiten einen systematischen Überblick zu geben.

Schlussfolgerung Unterschieden werden müssen Prozesse, die primär vom Neurokranium ausgehen, von sekundären Prozessen, die das Neurokranium infiltrieren. Für dieses wichtige diagnostische Merkmal ist die MRT meistens unverzichtbar, während die definitive Diagnose dann unter Berücksichtigung der Anamnese mit dem typischen Erscheinungsbild in der CT häufig gestellt werden kann. 


\section{Introduction}

There are generalized and focal diseases of the neurocranium with the latter being able to be further classified as multiple or solitary. Reactive, inflammatory, and tumorous processes are etiologically differentiated from metabolic disorders and systemic diseases which usually occur in a generalized or multiple manner. Solitary lesions of the neurocranium are often identified as asymptomatic incidental radiological findings.

Primary calvarial tumors comprise approx. $0.8 \%$ of all bone tumors [1], while secondary manifestations of a primary disease (e. g. metastases) or infiltrative processes from an adjacent region (e. g. meningiomas) are more common.

In the differential diagnosis it is often important to take into consideration whether there is a relationship to other anatomical compartments, such as the paranasal sinuses, the mastoids, the meninges, adjacent vascular structures or the galea.

Calvarial lesions are often detected as incidental findings on cranial magnetic resonance imaging (MRI) or computed tomography $(C T)$. Using $C T$, typical findings of classic diseases (e. g. hemangioma, osteoma, eosinophilic granuloma, and fibrous dysplasia) can be confidently diagnosed usually without additional imaging. Since the appearance on MRI is usually not particularly characteristic, a supplementary CT scan is often required.

While computed tomography can visualize bone destruction or a mineralized tumor matrix in particular [2], MRI is used due to the better soft tissue contrast for tissue characterization, spatial assignment to compartments, and visualization of an extraosseous component [3].

Classic skull X-rays are no longer used.

Venous or arterial cerebral MR and CT angiography or digital subtraction angiography (DSA) is used in primary vascular processes, infiltration of the large venous sinuses, or for treatment planning [4].

In the overview article, approx. $3500 \mathrm{CT} / \mathrm{MRI}$ scans of the cranium from the period $2008-2013$ were retrospectively evaluated by two experts (in radiology and neuroradiology) regarding processes in the neurocranium. Many of the lesions were histologically confirmed.

The goal of this overview article is to visualize typical solitary focal pathologies of the neurocranium and to provide a systematic overview of their locations, frequency, and radiological features on the basis of 12 entities ( $\triangleright$ Fig. $\mathbf{1}$ ).

For radiological diagnosis, lesions of the neurocranium should always be considered in connection with relevant clinical information. This includes patient age, clinical symptoms, and patient history regarding previous trauma, a primary disease and abnormal lab results. The evaluation of characteristic radiological features can help to narrow down the differential diagnoses [5].

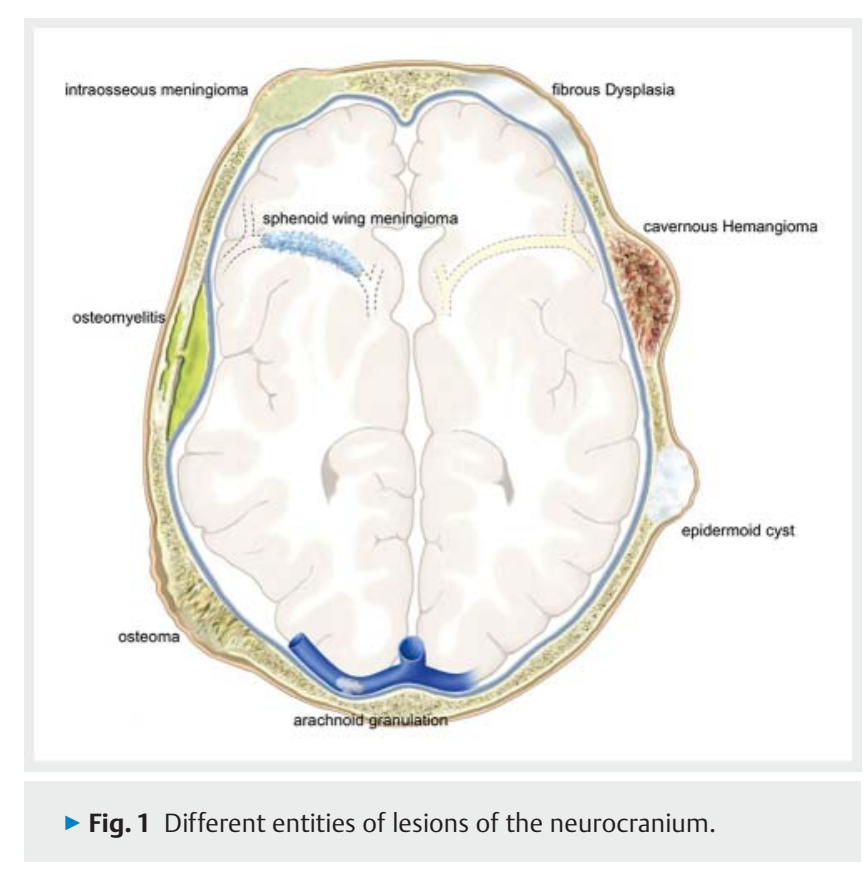

\section{Intraosseous meningioma}

\section{Definition and location}

Intraosseous meningiomas can be defined as neoplastic lesions with the greatest tumor portion located in bone with or without a moderate dural component.

Comprising $20 \%$ of all primary intracranial tumors, meningiomas are common entities usually located in the subdural space. In less than $1 \%$ of cases, meningiomas are extradural ectopic meningiomas in subcutaneous tissue, the main nasal cavities or paranasal sinuses, the orbital cavity, the salivary glands, or the diploe of the cranial bone [6]. In the latter case, they are referred to as intraosseous meningiomas [7]. Intraosseous meningiomas make up $14 \%$ of primary extradural meningiomas [8].

Etiologically, intrauterine displacement of meningocytes during the closure of midline structures, proliferation of meningocytes near sutures and the foramina, differentiation of Schwann cells into meningocytes and pluripotent mesenchymal cells are responsible for intraosseous meningiomas [9].

\section{Imaging}

CT usually shows an expansive osteoblastic lesion ( $\mathbf{F i g} . \mathbf{2 A - C}$ ) with enlargement of the bone. However, osteolytic or mixed (osteoblastic/osteolytic) subtypes of intraosseous meningioma have also been described in the literature [10]. The internal table can also be affected and has an irregular margin [11] ( $\triangleright$ Fig. 2DF). MRI signal behavior is variable on T2-weighted images and is usually isointense to hypointense in relation to the surrounding brain tissue on native T1-weighted images. The dura mater can also be involved in intraosseous meningiomas, depending on the duration of the disease. Contrast enhancement is seen in this case (dural tail). Intraosseous meningiomas are visualized in HE-staining in the histopathological specimen with isomorphic, meningo- 

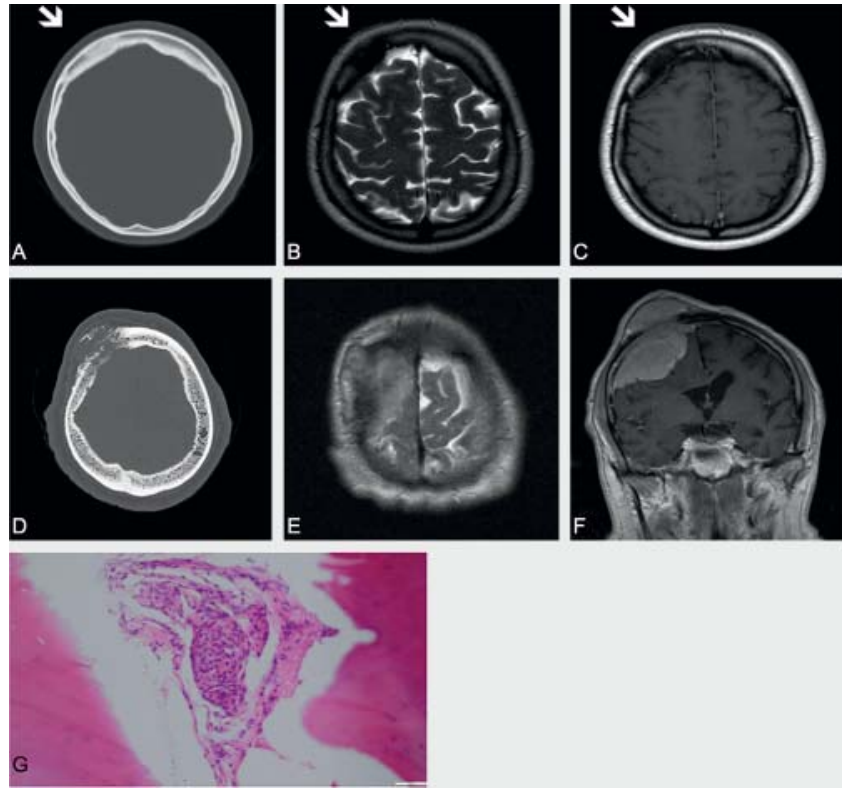

- Fig. 2 Intraosseous meningioma in a 40-year-old woman A-C. A Axial CT shows a focal osteoblastic lesion with no cortical bone destruction. B Axial T2-weighted image shows a hypointense lesion with no extraosseous component of the tumor. C Contrast-enhanced T1-weighted image shows no contrast enhancement. Meningioma in a 55-year-old woman $\mathbf{D}-\mathbf{F}$ with destruction of the adjacent bone $\mathbf{D}$ Axial T2-weighted image. E presents a large extraosseous tumor component with homogeneous enhancement after contrast media application. $\mathbf{F ~ H E}$-staining of the intraosseous meningioma. $\mathbf{G}$ shows isomorphic, meningothelial differentiated tumor without characteristics of atypia.

thelial differentiated tumor without characteristics of atypia ( $\triangleright$ Fig. 2G).

The first differential diagnosis to be considered is fibrous dysplasia. Osteoblastic metastases (primary disease usually known), Paget's disease and osteoma must also be considered. However, the internal lamina characteristically has a smooth border in the case of fibrous dysplasia and an irregular border in the case of intraosseous meningioma. Fibrous dysplasia tends to occur in childhood to early adulthood.

Bony infiltration of meningiomas primarily located in the subdural space must be differentiated from intraosseous meningiomas. This frequently results in significant erosive changes of the adjacent bone which complicates the differential diagnosis from a metastasis.

\section{Key points for clinical practice}

Intraosseous meningiomas are a rare entity that is usually asymptomatic and is typically seen on CT as an expansive osteoblastic lesion in adults. It can be difficult to differentiate from fibrous dysplasia or a solitary purely osteoblastic metastasis.

\section{Osteoma}

\section{Definition and location}

Osteomas are the most common benign tumor of the calvaria [12]. They are very slow growing bone tumors that can be diagnosed at any age but are most common in the 4th and 5th decade of life. However, the predilection sites are typically the paranasal sinuses, particularly the frontal sinus and the ethmoidal sinuses [13]. The majority of osteomas occur in the region of the cranial sutures. Osteomas typically occur at the external table ( $\triangleright$ Fig. 3A, B). When they arise from the internal table, they can be confused with a calcified meningioma.

In most cases, osteomas are asymptomatic incidental radiological findings. Osteomas of the paranasal sinuses can sometimes be caused by displacement of the excretory ducts, sinusitis or mucoceles. In the case of multiple osteomas, autosomal dominant congenital Gardner syndrome should be considered.

\section{Imaging}

On $\mathrm{CT}$, osteomas have density values almost identical to those of cortical bone since they are comprised of mature compact bone with a primarily lamellar structure ( $\triangleright$ Fig. $3 C$ ). A hypointense T1 signal, no contrast enhancement, and no soft tissue component are seen on MRI [14]. In the T2-weighted sequence, the signal behavior varies depending on the ratio of compact to spongy bone [4].

\section{Key points for clinical practice}

Osteomas have a density identical to that of cortical bone and arise primarily from the external table often in the region of the cranial sutures. No contrast enhancement and no soft tissue component are seen.

\section{Cavernous hemangioma}

\section{Definition and location}

As defined by the WHO, hemangiomas are benign lesions comprised of newly formed capillary or cavernous blood vessels. There is a histological difference between capillary and cavernous hemangiomas with cavernous hemangiomas being present in adults and capillary hemangiomas in children. Capillary hemangiomas are comprised of lobules of capillaries with some larger supply vessels, while cavernous hemangiomas are comprised of many large thin-walled spaces.

Primary intraosseous hemangiomas are hemangiomas that are normally located in the medullary cavity and occur most frequently in the vertebral body or the skull cap. These tumor-like lesions are slow growing and are usually asymptomatic.

\section{Imaging}

In the CT bone window, hemangiomas show a well-defined osteolytic lesion with the characteristic trabecular honeycomb pattern ( $\vee$ Fig. 4A, B) caused by thickened trabeculae bordering the 

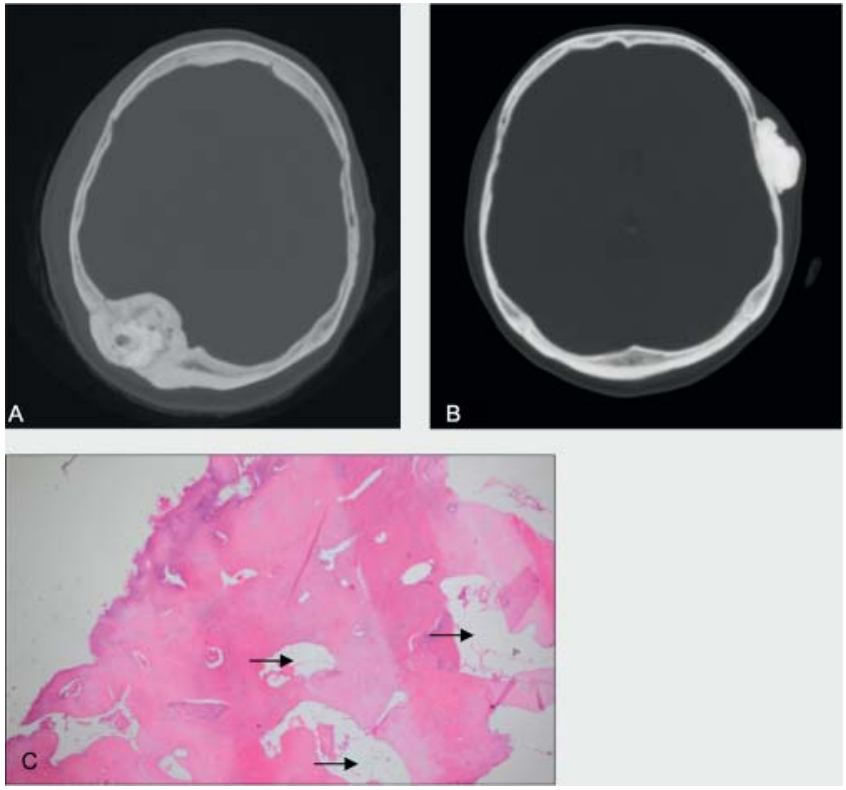

- Fig. 3 Osteoma of a 71-year-old woman. A Axial CT image on bone window settings showing a well-defined sclerotic tumor right occipital arising from the outer and inner cortical table. B Osteoma of a 45-year-old woman with axial CT image on bone window settings presenting an oval, dense sclerotic lesion related to the outer table of the left temporal bone. C HE-staining of the osteoma with compact, thickened lamellar bone; additional gap-like expanded fatty marrow is identified (arrows).

angiomatous channels. The fine cancellous bone trabeculae have disappeared and vertically oriented stringy lines of cancellous bone are clearly visible. The lesion originates in the diploe spaces and usually shows expansion of the external table. A periosteal reaction is also rarely seen [15].

After contrast administration, hemangiomas typically show significant enhancement [16]. However, this does not allow differentiation between capillary and cavernous hemangiomas.

On MRI hemangiomas are visualized with varying signal behavior due to remodeling processes after hemorrhage. On T1-weighted images, asymptomatic hemangiomas are usually hyperintense as a result of fatty atrophy with a stippled appearance due to the bony trabecula [17]. In contrast, atypical symptomatic hemangiomas have higher vascularity and less fat and therefore show a reduction in signal intensity on $\mathrm{T} 1$-weighted images.

On T2-weighted images, hemangiomas are hyperintense compared to the musculature due to the very slow flowing, at times stagnant, blood and the fat. The angiographic visualization of hemangiomas of bone can vary greatly. Some show typical corkscrew-like vessels ( $\boldsymbol{F i g . 4 C}$ ), while others are completely quiescent on angiography. Active hemangiomas can contain arteriovenous malformations. However, in principle, AV malformations are to be differentiated from hemangiomas since they are considered congenital vascular malformations.

CT and MRI images of a hemangioma are usually so typical that there are typically no problems regarding differential diagnosis. Only atypical hemangiomas can result in difficulties regarding
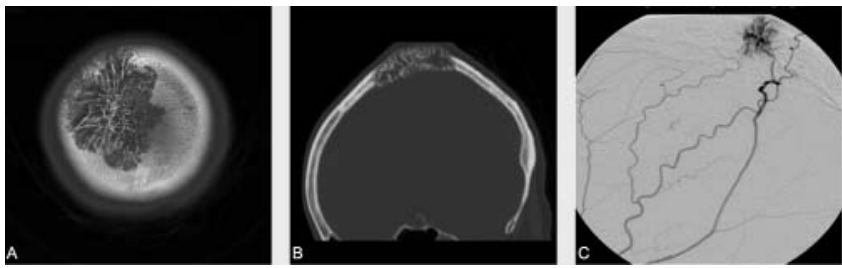

- Fig. 4 Cavernous hemangioma. A, B Axial and sagittal CT image on bone window settings from a 51-year-old woman showing welldefined expansive osteolytic lesion with thickened trabecula.

C Angiography of the cavernous hemangioma with hypervascularization.

the differential diagnosis due to the minimal amount of fat and the occasional clinical symptoms.

\section{Key points for clinical practice}

Cavernous hemangiomas are a relatively common incidental finding of an osteolytic lesion with the characteristic honeycomb trabecular pattern. This vascular tumor-like lesion has a typical appearance on T1-weighted images due to the high amount of fatty atrophy and is usually asymptomatic. In contrast, atypical symptomatic hemangiomas have higher vascularity and less fat and therefore show a reduction in signal intensity on $\mathrm{T} 1$-weighted images.

\section{Epidermoid cyst}

\section{Definition and location}

Epidermoid cysts are the result of a dysontogenetic developmental disorder in which the entrapment of ectodermal elements results in a non-neoplastic mass containing an epidermis-like cyst wall and keratin lamellae. These cysts are usually caused by incomplete cell division of the neural tissue from the cutaneous external germ layer in the 3 rd to 5 th week of gestation but can also be caused in rare cases by trauma or surgery [18]. Epidermoid cysts are usually asymptomatic and first appear in adulthood due to their protracted linear growth [4]. Epidermoid cysts occur most commonly in the cerebellopontine angle (37\%), the parasellar region (30\%), and less commonly also in the middle cranial fossa [19]. However, approximately $10 \%$ of epidermoid cysts are also extradural and usually intraosseous (within the diploe). In the histopathological specimen, HE-staining demonstrates parts of an epidermoid cyst with a keratinized epithelium and keratin lamellae ( $\triangleright$ Fig. 5D). Both the epithelial cyst wall and the keratin lamellae express the pan-cytokeratin marker AE1/3 ( Fig. 5E).

\section{Imaging}

On CT, epidermoid cysts are visualized as hypodense, lobulated, osteolytic masses in a typical location and usually have a smooth border with thinning and more rarely rupture of the internal and external table [1] ( $\vee$ Fig. 5A). The density values of the lesion are equivalent to those of liquid in $\mathrm{T} 1$ and $\mathrm{T} 2$ weighting but can vary depending on protein content and bleeding [20] ( $\mathbf{F i g . 5 B}, \mathbf{C}$ ). 

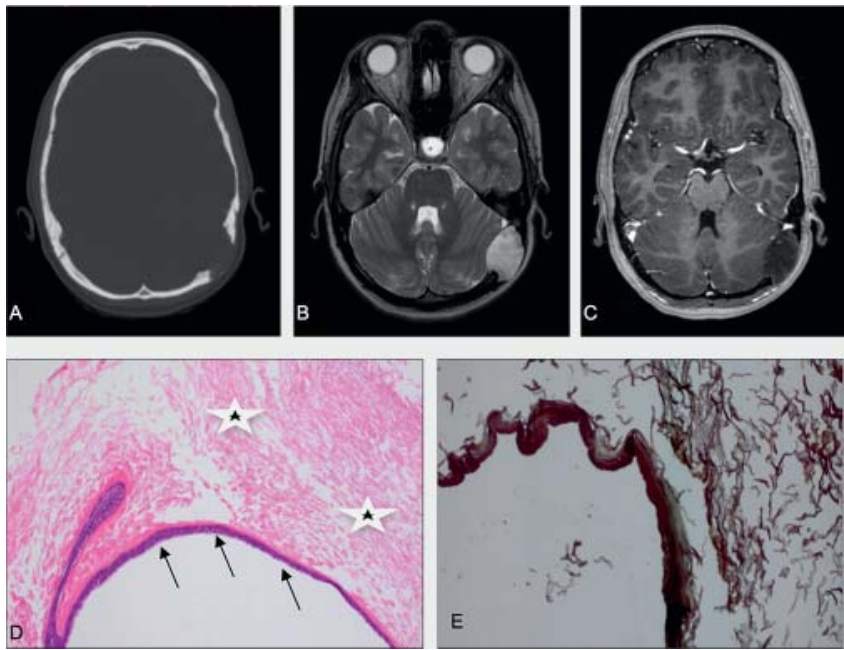

- Fig. 5 A Axial CT image on bone window settings. B Axial T2weighted image showing a left parietal well-defined expansive osteolytic lesion in a 19-year-old woman with thinning and breach of the cortical bone. The content is predominantly high signal on T2. C Axial contrast-enhanced T1-weighted MR image shows no contrast enhancement. D HE-staining demonstrates parts of the epidermoid cyst with keratinizing epithelium (arrows) and keratinized epithelial cells (stars). E AE 1/3-staining: Both the epithelial cyst wall and keratinized epithelial cells that express the pan-cytokeratin marker $\mathrm{AE} 1 / 3$.

Calcifications are sometimes also seen within the lesion. A narrow enhanced border that probably corresponds to an inflammatory response in the periphery is sometimes also seen in epidermoid cysts after contrast administration. A hyperintense FLAIR and DWI signal and a correspondingly reduced ADC value are characteristic on the b1000 MRI image.

\section{Key points for clinical practice}

Epidermoid cysts are usually asymptomatic and first appear in adulthood. A hyperintense FLAIR and DWI signal and a correspondingly reduced ADC value are characteristic on the b1000 MRI image.

\section{Arachnoid granulations}

\section{Definition and location}

Arachnoid granulations are villiform formations of the arachnoid membrane that protrude into the large intracranial venous sinuses. They appear in the superior sagittal sinus, transverse sinus, cavernous sinus, and sigmoid sinus in decreasing order of frequency.

They can expand thereby compressing adjacent bone and cause osteolysis, then sometimes resulting in problems regarding the differential diagnosis with respect to calvarial metastases, particularly in the case of patients with corresponding previously known neoplasias. In addition, circumscribed thrombus in the transverse sinus is a recurring differential diagnosis. They can rarely become very large ( $\triangleright$ Fig. $\mathbf{6}$ ) and can be confused with a mass.
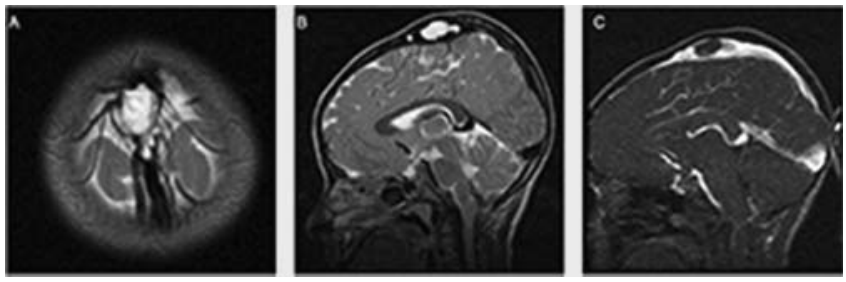

- Fig. 6 6-year-old boy with giant arachnoid granulation. A, B Axial and sagittal T2-weighted images present a hyperintense signal of the arachnoid granulation at the superior sagittal sinus and cortical thinning of the adjacent skull but no osteolysis. C Venous MR angiography shows a large contrast defect.

\section{Imaging}

Arachnoid granulations are usually not hyperdense on CT and the adjacent osteolysis has a smooth border without increased sclerosis. They are typically hyperintense on T2w MRI images.

In addition, the typical MRI signs of a thrombus are an isointense to hyperintense extensive signal in T1 weighting and a significant signal reduction in $\mathrm{T} 2 * /$ heme sequences. The main features of a large arachnoid granulation are non-contrastenhancing granula with central, linear enhancement and surrounding signal-intensive blood on contrast-enhanced CT or MR venography.

\section{Key points for clinical practice}

Arachnoid granulations can sometimes be very large and cause osteolytic changes to adjacent bone. They must be differentiated from sinus and cerebral vein thrombosis and osteolysis caused by a neoplasia.

\section{Fibrous dysplasia}

\section{Definition and location}

Fibrous dysplasia is an ossification disorder caused by a mutation and is a non-neoplastic developmental disorder of the bone-forming mesenchymal tissue with incorrect differentiation into mature bone. Nevertheless, it is classified in newer studies as benign, nonencapsulated neoplasias [21].

Fibrous dysplasia mainly occurs in childhood and early adulthood and can spontaneously resolve after puberty. To date, individual cases of osteosarcomatous, fibrosarcomatous and chondrosarcomatous degeneration have been observed, with the probability of degeneration being specified as approx. $0.4 \%$ [22, $23]$. According to the literature, fibrous dysplasia is located on the cranial bones in $15 \%$ of cases, primarily in the region of the paranasal sinuses and the jaw [24]. It can be monostotic or rarely polyostotic in various syndromes that typically manifest in childhood (e.g. McCune-Albright) [25].

Symptoms can result from the compression of adjacent structures. Compression of the cranial nerves can occur particularly in craniofascial dysplasia. 


\section{Imaging}

Depending on the distribution of the connective tissue and bone, computed tomography shows a honeycomb-cystic pattern with the characteristic, ground glass appearance ( $\vee$ Fig. 7A). The bone typically appears enlarged and ballooned with a thinned cortical bone. The external table is affected more than the internal table [26]. Speckled calcifications also occur in some cases. The coexistence of different changes is typical of fibrous dysplasia but can result is problems regarding differential diagnosis on MRI and can resemble aggressive lesions. On T2w images, lesions can appear very hypointense or also extremely hyperintense depending on the relationship between fibrous tissue and bony matrix [27]. After contrast administration, enhancement with low signal intensity tends to be characteristic for these lesions but intense enhancement is also possible [1] ( $\mathbf{F i g} . \mathbf{7 B}, \mathbf{C}$ ).

The diagnosis of fibrous dysplasia can be confidently made in most cases due to its characteristic appearance on CT. In addition, the intactness of the cortical bone can be evaluated on CT to differentiate from a malignant tumor.

\section{Key points for clinical practice}

Fibrous dysplasia is a typical bone lesion in children and adolescents. The diagnosis of fibrous dysplasia can be confidently made in most cases due to its characteristic ground-glass appearance on $\mathrm{CT}$.

\section{Osteomyelitis}

\section{Definition and location}

Osteomyelitis is bone inflammation usually caused by bacteria. Osteomyelitis of the calvaria, which is very rare, is usually a secondary disease. It is often caused by an injury or an inflammatory process in the paranasal sinuses, the mastoids, or the orbital cavity. Osteomyelitis can also rarely occur after neurosurgical interventions. This can occur 1 to 12 months after an intervention [28] and is usually caused by Staphylococcus aureus [29]. Purulent osteomyelitis is acute and chronic. In the initial stage of inflammation, the bacteria trigger a circumscribed inflammatory response resulting in localized cell death and ultimately in circumscribed osteonecrosis. Over time, the infection becomes demarcated with a margin of granulation tissue and new bone.

\section{Imaging}

Osteomyelitis of the calvaria results in focal osteolysis with an unclear border with a moth-eaten appearance in some cases and rarely also with formation of a sequestrum. Chronic courses are also associated with a reaction of the adjacent bone in the form of increased sclerosis in the surrounding area. Sometimes the findings are only very minor without major concomitant extraosseous findings ( $>$ Fig. 8).

Hyperintense edema is often seen in the acute type and increased sclerosis is seen in the chronic type on T2-weighted MRI. An extraosseous soft tissue component can be effectively visualized on MRI. Edema and significant contrast enhancement
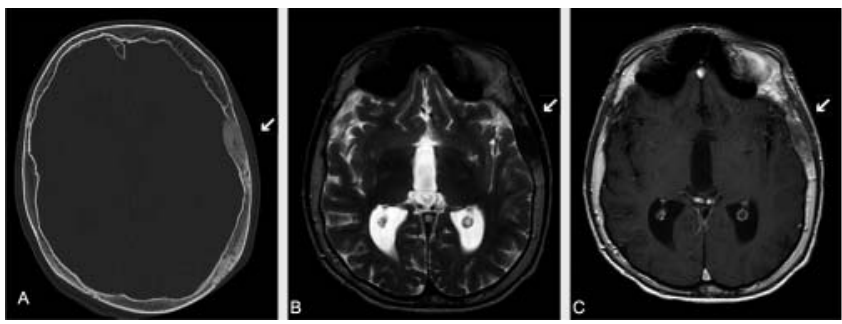

- Fig. 7 Fibrous dysplasia in a 20-year-old man. A Axial CT image shows an expansive intradiploic fronto-temporal lesion with the typical "ground glass" appearance. B Axial T2-weighted image presents a low signal intensity lesion. C Axial, contrast-enhanced T1weighted image shows areas with and without contrast enhancement.
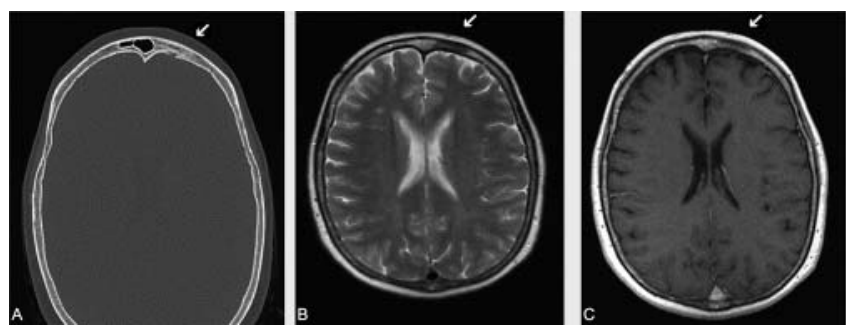

- Fig. 8 Osteomyelitis in a 52-year-old woman. A Axial CT image shows a moth-eaten destruction of the inner table in the left frontal bone. B T2-weighted images show a hypointense lesion. C Contrastenhanced T1-weighted images present low to intermediate contrast enhancement and no empyema.

are typically seen in acute osteomyelitis, while mild contrast enhancement can often be seen in chronic cases.

\section{Key points for clinical practice}

Osteomyelitis of the calvaria is very rare and is usually a secondary disease. It is often caused by an injury or an inflammatory process. It results in focal osteolysis with a moth-eaten appearance in some cases. In the chronic type, it results in increased sclerosis in the surrounding area, rarely also in the formation of a sequestrum.

\section{Langerhans cell histiocytosis}

\section{Definition and location}

Langerhans cell histiocytosis (LHC) is a systemic disease of unclear etiology. However, an intercellular communication defect with a cytokine imbalance between T-cells and Langerhans cells is under discussion. Eosinophilic granulomas are the most common and mildest form [30]. LHC is a generic term for a group of systemic diseases with different courses and proliferation of dendritic Langerhans cells and ontogenetically related cell variants [26, 31]. In general, the disease can affect any organ. However, bone, particularly the calvaria, is most commonly affected [32].

The clinical picture is highly variable and is characterized by the different organ manifestations. Patients can experience local pain in the calvaria and have a palpable, growing lesion [33]. However, 
there are also patients who are asymptomatic and are diagnosed on the basis of an incidental finding.

LHC can occur at any age but is most common in children and adolescents [32] with the peak age being in the first three years of life [34].

The lesion originates in the diploe and extends through the internal table and the external table. The parietal bone is most commonly affected [35].

In particular, the disease must be differentiated from non-Langerhans histiocytosis (class II histiocytosis). The prognosis for localized Langerhans cell histiocytosis is very good. In multisystemic $\mathrm{LCH}$, the prognosis depends on the age of the patient and primarily on the involvement of organs at risk and the response to treatment.

\section{Imaging}

On computed tomography, the appearance depends on the type and phase of the disease. The calvarial lesions appear quickly in the early stage of the disease. Imaging shows a clearly demarcated osteolytic lesion without a sclerotic border or periosteal reaction. Slanted edges are often seen due to the unequal involvement of the internal table and external table [36]. A central bone sequestrum is a characteristic finding on CT ( $>$ Fig. 9A). However, this sequestrum is also described in other diseases, e. g. in osteomyelitis, metastases, and epidermoid cysts [37]. After contrast administration, eosinophilic granulomas can demonstrate reactive dural ("dural tail sign") and galeal contrast enhancement [38] ( $\triangleright$ Fig. 9C, D). The lesions are hypointense on T1-weighted MRI and hyperintense on T2-weighted MRI [39] ( $>$ Fig. 9B). A reduced signal in T2-weighted sequences is described as a sign of healing [12].

The diagnosis is typically verified by biopsy of affected tissue with histopathological and immunohistochemical testing.

\section{Key points for clinical practice}

Langerhans cell histiocytosis is a systemic disease in children and adolescents. Imaging typically shows osteolysis with a soft tissue component. The diagnosis is typically confirmed by a biopsy.

\section{Paget's disease}

\section{Definition and location}

Paget's disease of bone is characterized by localized high-grade increased bone remodeling triggered by pathological giant osteoclasts with significantly increased resorption activity [40]. Three phases of the disease (lytic, mixed, sclerotic) are known and can occur in the same bone [41]. The result of this excessive bone remodeling accounts for the characteristic mosaic pattern $(\triangleright$ Fig. 10A, B). Paget's disease is slow progressing and mainly occurs in older people.

A malignant change of the bone in Paget's disease is a rare complication.

Involvement of the calvaria is possible. Monostotic and polyostotic forms are known. Approximately $10 \%$ of all patients experi-
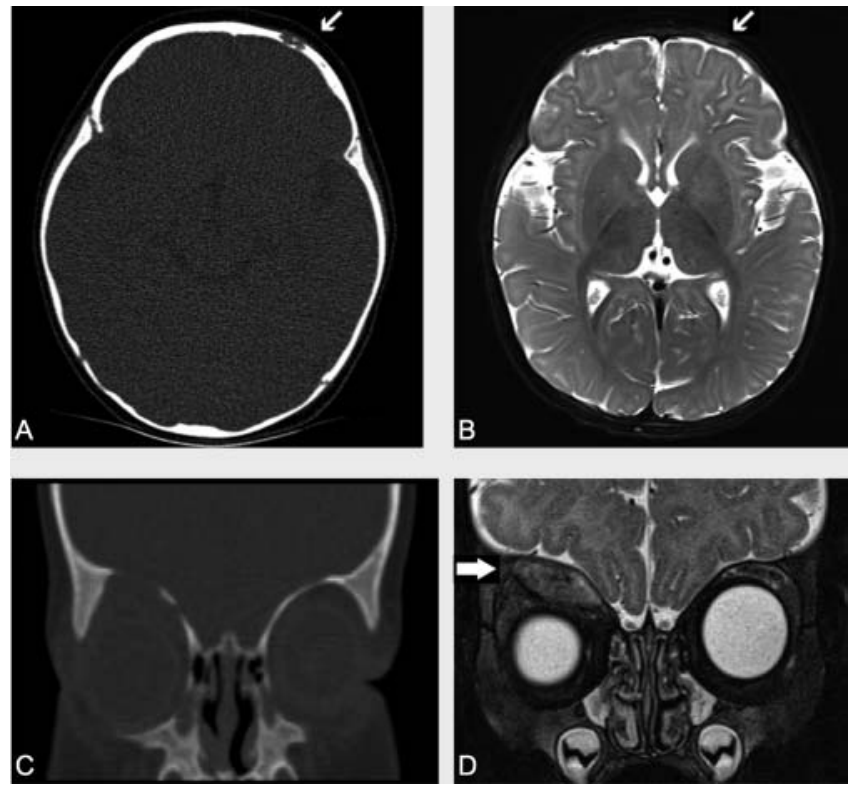

- Fig. 9 Langerhans cell histiocytosis in a 6-month-old boy. A Axial CT image shows a well-defined "punched-out" osteolytic lesion in the left frontal bone with the central "button sequestrum". B Axial T2-weighted image presents a dural T2-hyperintense lesion. Another manifestation of Langerhans cell histiocytosis in the same patient. $\mathbf{C}$ shows a bone defect right supraorbital and $\mathbf{D}$ shows presentation of supraorbital soft tissue component.
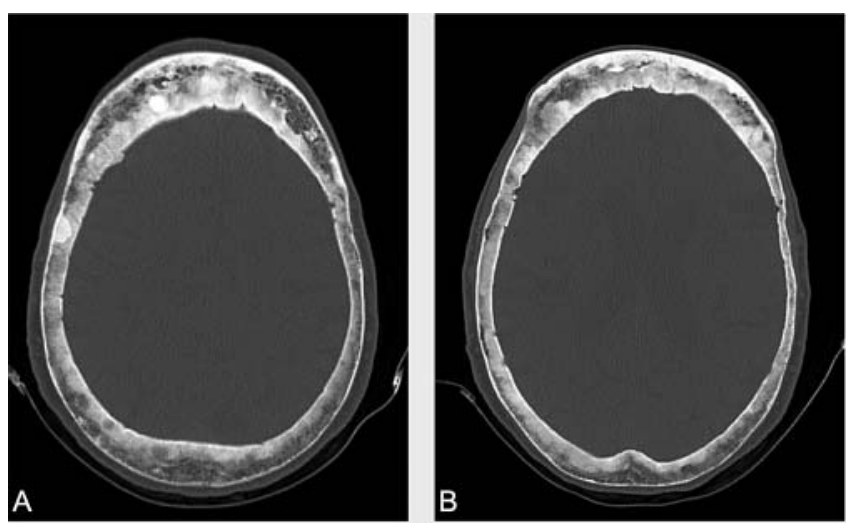

- Fig. 10 Paget's disease in a 59-year-old man. A, B Axial CT images on bone window settings show bony expansions with focal sclerotic areas.

ence symptoms (pain). The uncontrolled bone formation can result in nerve compression particularly of the cranial nerves that can cause sensorineural hearing loss. Conduction deafness due to bony joint bridging of the auditory ossicles has also been observed.

\section{Imaging}

The characteristic appearance on CT includes bony enlargement or coarsening of the trabeculae and cortical thickening [2]. In the osteolytic phase, transparent calvarial lesions with a smooth border can be detected. These can expand beyond the sutures and 
occur most frequently in the frontal bone and occipital bone. In the mixed phase (lytic/sclerotic), the lesions often have a honeycomb-like appearance with accompanying thickening of the calvaria [36] ( Fig. 10A, B).

T1-weighted MRI shows hypointense areas in the diploe caused by the fatty marrow being replaced by fibrovascular tissue. Significant enhancement is seen after contrast administration.

\section{Key points for clinical practice}

Paget's disease is a slowly progressing disease mainly occurring in older people and is usually observed in the frontal bone and occipital bone of the the calvaria. Three phases of the disease are known with the combination of lysis and sclerosis being observed most frequently. This excessive bone remodeling results in the characteristic mosaic pattern.

\section{Bone metastases}

\section{Definition and location}

Metastases are the most common malignant bone lesions in adults [42]. Almost any malignant tumor can cause metastases in the calvaria. However, breast cancer is most frequently associated with metastatic calvarial lesions [43]. There are osteolytic, osteoblastic, and mixed manifestations [12] that can also occur on a singular basis. Pulmonary, gastrointestinal, and urogenital primary tumors are primarily seen in men, while breast, lung, and gastrointestinal tumors and melanomas are most commonly seen in women. Hemorrhagic metastases are primarily observed in melanoma, bronchogenic carcinoma, and breast cancer as well as in renal cancer and choriocarcinoma as the primary tumor. Combined osteolytic and osteoblastic metastases are primarily seen in lung cancer and breast cancer. Calvarial metastases rarely cause symptoms. However, pain and loss of cerebral nerve function have been observed in metastases of the base of the skull. Dural metastases can act as regional masses or infiltrate adjacent brain structures. Calvarial metastases are most common in neuroblastomas in children [44]. It is important to remember that a singular calvarial metastasis can sometimes be the only evidence of a metastatic disease. This is the case in particular in renal cell carcinoma or thyroid carcinoma.

\section{Imaging}

CT effectively shows the extent of bone destruction ( $\triangleright$ Fig. 11A) and irregular borders are often seen [43] ( $\triangleright$ Fig. 11B). MRI using fat-suppressed $\mathrm{T} 1$ sequences is particularly suitable for visualizing small lesions and the extent of spread intracranially or within the diploe. Contrast enhancement is typically seen in metastases [43].

\section{Key points for clinical practice}

Almost all malignant tumors can result in calvarial metastases. Breast cancer in women and prostate cancer in men are most commonly associated with metastatic bone lesions. Patient history and patient age must be taken into consideration in the differential diagnosis.
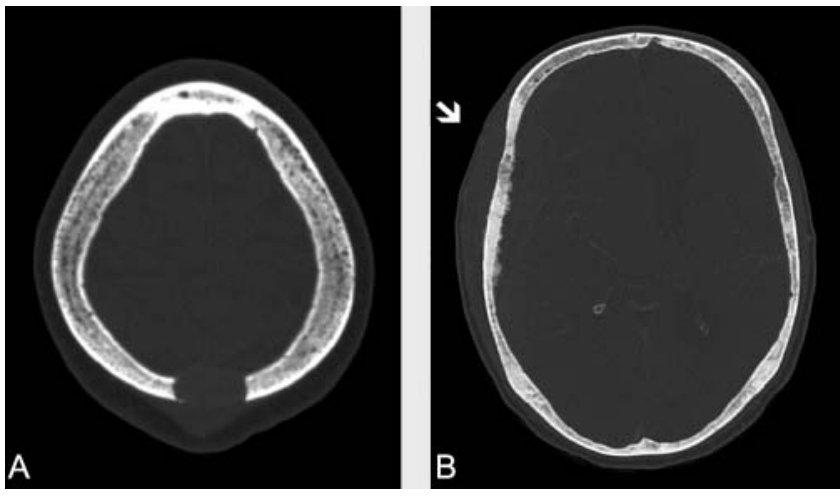

- Fig. 11 A Axial CT on bone window settings from a 73-year-old woman with solitary osseous metastasis shows osteolytic destruction of the occipital bone with a soft-tissue mass. B Axial CT on bone window settings from a 51-year-old man with osteoblastic metastasis demonstrates a sclerotic bone lesion of the right temporal bone with spiculae.

Plasmacytoma/multiple myeloma and systemic lymphoma with bone marrow involvement and primary bone lymphoma are discussed in detail below.

\section{Plasmacytoma/multiple myeloma}

In the case of solitary plasmacytoma, abnormal plasma cells in the bone marrow accumulate and form a bone or soft tissue tumor (extramedullary). The involvement of the calvaria can be diffuse or multifocal in the case of multiple myeloma and focal and singular in plasmacytoma.

Lytic defects with a clear margin and a "punched out" appearance are typically seen in plasmacytoma and multiple myeloma on CT. MRI is very sensitive with respect to the detection of lesions that are typically hypointense on $\mathrm{T} 1$ and hyperintense on $\mathrm{T} 2$ and show contrast enhancement. The so-called "salt and pepper pattern" is the result of diffuse infiltration of the bone marrow [45].

\section{Key points for clinical practice}

Lytic defects with clear margins and a "punched out" appearance are typically seen. The "salt and pepper pattern" resulting from diffuse infiltration of the bone marrow is characteristic on MRI.

\section{Lymphoma}

Primary bone lymphoma is rare [46]. The imaging characteristics are nonspecific. Bone destruction can be visible on computed tomography. However, a permeative growth pattern without a tumor matrix and marginal sclerosis ( $\triangleright$ Fig. 12A) with an intracranial and/or extracranial soft tissue component ( $\vee$ Fig. 12B) is more common [47]. Leptomeningeal metastases can be missed on CT. Lymphomas can be slightly hyperintense on native T1w MRI in the case of a high cell density but can also be hypointense. Lymphomas tend to demonstrate a hypointense signal on T2. After contrast administration, lymphomas show significant con- 

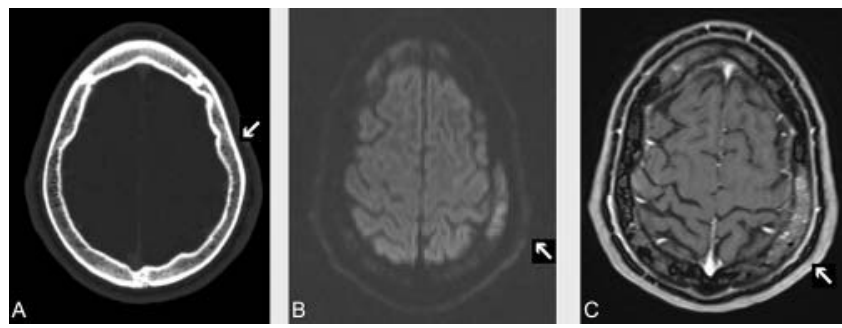

- Fig.12 A Axial CT on bone window settings from an 11-year-old boy with lymphoma shows no detectable bone involvement of the left parietal bone. B Axial MRI demonstrates high signal intensity in DWI in the left parietal bone with strong enhancement $\mathbf{C}$.

trast enhancement ( $\bullet$ Fig. 12C) and sometimes a decreased ADC due to the high cell density.

Involvement of the bone marrow is more common that primary bone lymphoma in systemic lymphoma. However, there are no major differences with respect to imaging.

\section{Key points for clinical practice}

Primary bone lymphoma is rare. A permeative growth pattern without a tumor matrix and marginal sclerosis with an intracranial and/or extracranial soft tissue component is often seen. After contrast administration, significant contrast enhancement is usually seen and a decreased ADC is sometimes seen due to the high cell density. Secondary lymphoma of the bone marrow is more common that primary bone lymphoma.

\section{Conclusion}

There are a number of neoplastic and non-neoplastic solitary lesions of the neurocranium that are often diagnosed as an incidental radiological finding. It is necessary to differentiate between primary osseous lesions that originate from the neurocranium and secondary processes that infiltrate the neurocranium. MRI is often essential for this differentiation, and diagnosis can then be made based on the characteristic appearance on $\mathrm{CT}$ in connection with patient history and patient age. Osteolytic lesions, such as metastases, osseous lymphoma, osteomyelitis, epidermoid cysts, and Langerhans cell histiocytosis, must be differentiated from osteoblastic lesions, such as metastases, fibrous dysplasia, osteoma, and intraosseous meningioma.

\section{Conflict of Interest}

The authors declare that they have no conflict of interest.

\section{References}

[1] Lloret I, Server A, Taksdal I. Calvarial lesions: A radiological approach to diagnosis. Acta Radiol 2009; 50: 531 - 542

[2] Garfinkle J, Melancon D, Cortes M et al. Imaging pattern of calvarial lesions in adults. Skeletal Radiol 2011; 40: 1261-1273
[3] Amaral L, Chiurciu M, Almeida JR et al. MRI Imaging for evaluation of lesions of the cranial vault: a pictorial essay. Arq Neuropsiquiatr 2003; 61: $521-532$

[4] Ortiz O, Schochet S, Bastug D. Imaging evaluation and clinicopathologic correlation of mass lesions involving the calvaria. Part I: congenital and traumatic lesions. Int J Neuroradiol 1999; 5: 96-108

[5] Miller TT. Bone tumors and tumorlike conditions: analysis with conventional radiography. Radiology 2008; 246: 662 -674

[6] Lang FF, Macdonald K, Fuller GN et al. Primary extradural meningiomas: a report of nine cases and review of the literature from the era of computerized tomography scanning. J Neurosurg 2000; 93: 940-950

[7] Pfatschenbacher A. Intraosseus meningioma: a case report and differential diagnosis. Fortschr Röntgenstr 2000; 172: 303 - 304

[8] Daffner RH, Yakulis R, Maroon JC. Intraosseous Meningioma. Skeletal Radiol 1998; 27: 108-111

[9] Spraul CW, Gareis O, Lang GK. Primary extradural meningioma of the orbits: a report of a patient and review of the literature. Klin Monatsbl Augenheilkd 1996; 209: 322 - 327

[10] Elder JB, Atkinson R, Zee Ch-S et al. Primary intraosseous meningioma. Neurosurg Focus 2007; 23: 1 - 9

[11] Arana E, Diaz C, Latorre FF et al. Primary intra-osseous meningiomas. Acta Radiol 1996; 37: 937 -942

[12] Arana E, Marti-Bonmati L. CT and MRI Imaging of focal calvarial lesions. Am J Roentgenol 1999; 172: $1683-1688$

[13] Oostvogel CW, Hüttenbrink KB. Pathogenesis of osteoma of the skull. Laryngorhinootologie 1991; 70: 87-89

[14] Avrahami I, Even I. Osteoma of the inner table of the skull. Clin Radiol 2000; 55: $435-438$

[15] Murphey MD, Fairbairn KJ, Parman LM et al. Musculoskeletal angiomatous lesions: radiologic-pathologic correlation. Radiographics 1995; 15: $893-917$

[16] Politi M, Romeike BFM, Papanagiotou P et al. Intraosseous hemangioma of the skull with dural tail sign: radiologic features with pathologic correlation. Am J Neuroradiol 2005; 26: 2045-2052

[17] Bastug D, Ortiz O, Schochet S. Haemangiomas in the calvaria: imaging findings. Am J Roentgenol 1995; 164: 683-687

[18] Smirniotopuolos JG, Chiechi MV. Teratomas, dermoides and epidermoides of head and neck. Radiographics 1995; 15: 1437 - 1455

[19] Hanft SJ, Komotar RJ, Raper DM et al. Epidermoid tumors of the temporal lobe as epileptogenic foci. J Clin Neurosci 2011; 18: 1396 - 1399

[20] Arana E, Latorre FF, Revert A et al. Intradiploic epidermoid cysts. Neuroradiology 1996; 38: $306-311$

[21] Lisle DA, Monsour PA, Maskiell CD. Imaging of craniofacial fibrous dysplasia. J Med Imaging Radiat Oncol 2008; 52: 325-332

[22] Ozaki T, Lindner N, Blasius S. Dedifferentiated chondrosarcoma in Albright syndrome. A case report and review of the literature. J Bone Joint Surg Am 1997; 79: 1545-1551

[23] DiCaprio MR, Enneking WF. Fibrous dysplasia. Pathophysiology, evaluation and treatment. J Bone Joint Surg Am 2005; 87: 1848 - 1864

[24] Fechner RE, Mills StE (Hrsg) Tumor of the bones and Joints. Atlas of tumor pathology; Am Reg Pathol; 1992: 147-151

[25] Willatt JMG, Quaghebeur G. Calvarial masses of infants and children. A radiological approach. Clin Radiol 2004; 59: 474-486

[26] Chung EM, Murphey MD, Specht CS et al. Pediatric orbit tumors and tumorlike lesions: osseous lesions of the orbit. Radiographics 2008; 28: $1193-1214$

[27] Jee W-H, Choi K-H, Choe B-Y. Fibrous dysplasia: MRI imaging characteristics with radiopathologic correlation. Am J Roentgenol 1996; 167: $1523-1527$

[28] Ryu J, Cheong JH, Kim JH et al. Delayed infection following cranioplasty. Kor Neurotraumatol Soc 2005; 1: 110-113 
[29] Davies BM, Jones A, Patel HC. Implementation of a care bundle and evaluation of risk factors for surgical site infection in cranial neurosurgery. Clin Neurol Neurosurg 2016; 144: 121-125

[30] Carter RMS, Anslow P. Imaging of the Calvarium. Semin Ultrasound CT MRI 2009; 30: 465-491

[31] Kumar YP, Agrawal ], Mohanlakshmi ] et al. Langerhans cell histiocytosis revisited: Case report with review. Contemp Clin Dent 2015; 6: 432 436

[32] Azouz EM, Saigal G, Rodriguez MM et al. Langerhans' cell histiocytosis: pathology, imaging and treatment of skeletal involvement. Pediatr Radiol 2005; 35: $103-115$

[33] Okamoto K, Ito ], Furusawa T et al. Imaging of calvarial eosinophil granuloma. Neuroradiology 1999; 41: $723-728$

[34] Leonidas JC, Guelfguat M, Valderrama. Langerhans' cell histiocytosis. Lancet 2003; 361: $1293-1295$

[35] David R, Oria RA, Kumar R et al. Radiologic features of eosinophilic granuloma of bone. Am J Roentgenol 1989; 153: 1021 - 1026

[36] Mitra I, Duraiswamy M, Benning J et al. Imaging of focal calvarial lesions. Clin Radiol 2016; 71: $389-398$

[37] Krasnokutsky M. The button sequestrum sign. Radiology 2005; 236: $1026-1027$

[38] Guermazi A, Lafitte F, Miaux Y et al. The dural tail sign d beyond meningioma. Clin Radiol 2005; 60: $171-188$
[39] Yalcin O, Yildirim T, Kizilkilic O et al. CT and MRI findings in calvarial noninfectious lesions. Diagn Interv Radiol 2007; 13: 68-74

[40] Hosking D, Meunier PJ, Ringe JD et al. Paget's disease of bone: diagnosis and management. Brit Med J 1996; 312: 491 -494

[41] Cortis K, Micallef K, Mizzi A. Imaging Paget's disease of bone d from head to toe. Clin Radiol 2011; 66: 662-672

[42] Mitsuya K, Nakasu Y, Horiguchi S et al. Metastatic skull tumours: MR features and a new conventional classification. J Neurooncol 2011; 104 : $239-245$

[43] Stark AM, Eichmann T, Mehdorn HM. Skull metastases: clinical features, differential diagnosis, and review of the literature. Surg Neurol 2003; 60: $219-226$

[44] Egelhoff JC, Zalles C. Unusual CNS presentation of neuroblastoma. Pediatr Radiol 1996; 26: 51 - 54

[45] Baur-Melnyk A, Buhmann S, Durr HR et al. Role of MRI for the diagnosis and prognosis of multiple myeloma. Eur J Radiol 2005; 55: 56-63

[46] Duyndam DAC, Biesma DH, Van Heesewijk JPM. Primary non-Hodgkin lymphoma of the cranial vault: MRI features before and after treatment. Clin Radiol 2002; 57: 948 - 950

[47] Kantarci M, Erdem T, Alper F et al. Imaging characteristics of diffuse primary cutaneous B-cell lymphoma of the cranial vault with orbital and brain invasion. Am J Neuroradiol 2003; 24: 1324-1326 